\title{
Accepting the Internet-of-Things in our homes: The role of user skills
}

\begin{abstract}
The present study investigates whether skills associated with operating the Internet-of-Things (IoT) contribute to the acceptance and (intended) usage of IoT technology and investigates to what extent these IoT skills are influenced by Internet skills. Extending the technology acceptance model (TAM), we conducted an online survey among 1,356 respondents, a sample representative of the Dutch population. The results revealed that IoT skills directly contribute to IoT use and testify to the relevance of the TAM for predicting IoT acceptance and usage. However, people's attitudes towards the IoT did not influence its acceptance, suggesting that users are not yet fully aware of how IoT use affects their privacy and quality of life in general. Furthermore, the results revealed that several Internet skills are an important precedent for IoT acceptance and usage. Mobile, information navigation, social, and creative Internet skills directly or indirectly contributed to the level of IoT skills. It can be concluded that people's assessments of their IoT skills and that pragmatic considerations are important for its acceptance and that being skilled in using the IoT requires sufficient Internet skills.
\end{abstract}

Keywords: Internet-of-Things, Internet skills, IoT skills, technology acceptance, digital divide, digital inequality

\section{Introduction}

Over the years, the nature of Internet use has become more complex as the Internet has developed from Web 1.0 to Web 4.0. In Web 1.0, users were limited to reading content, whereas through the introduction of Web 2.0, they were enabled to contribute to the Web by creating, storing, and sharing this content themselves. Web 3.0 goes further by introducing semantics, facilitating smoother communication between humans and machines. Web 3.0 moves away from a connection between data and knowledge, using keywords and tags to a connection based on natural language and intrinsic meanings. This development was initiated to improve information searching and data sharing. Although Web 3.0 is still under development, the era of Web 4.0 has arrived. Web 4.0 involves a connection with the Web anytime and anywhere, personalized services via the use of continuously obtained data, and a continuous connection with other users. These characteristics of Web 4.0 can be referred to as ubiquity, identity, and connection, respectively (Noh, 2015).

One of the new technological developments that implies objectification of the three characteristics of Web 4.0 - ubiquity, identity, and connection - is that of the Internet-of-Things (IoT). Although there is no generally accepted definition of the IoT (Whitmore, Agarwal, \& $\mathrm{Da} \mathrm{Xu,} \mathrm{2015),} \mathrm{based} \mathrm{on}$ 
communalities (e.g., Atzori, Iera, \& Morabito, 2010; Gubbi, Buyya, Marusic, \& Palaniswami, 2013; Perera, Zaslavsky, Christen, \& Georgakopoulos, 2014), Van Deursen and Mossberger (2018, p. 4) consider the IoT as "systems that (1) contain ubiquitous "everyday" objects that are accessible through the Internet and equipped with sensing, storing, and processing capabilities that allow these objects to understand their environments; (2) contain identifying and networking capabilities that allow them to communicate information about themselves; (3) involve object-object, object-person, and person-person communication; and (4) make autonomous decisions".

Together, these IoT characteristics change the nature of Internet use. Technology acceptance is a first step to beneficially using the IoT. Once accepted, the IoT potentially offers several benefits as it enables individuals to make better decisions (Van Deursen \& Mossberger, 2018). The data autonomously gathered by IoT devices could, for example, support informed decision making concerning the use of energy or health practices. These decisions could result in cost savings and increased living convenience. In terms of energy-related use, this could be translated to lower energy costs and, for instance, a personalized heating plan regulating room temperature. For health practices, Web 4.0-based decisions could imply lower healthcare costs, personalized health insurance, and personalized care. An example of such personalized care could be being monitored remotely over the Internet, minimizing the number of doctor visits.

However, the introduction of Web 4.0 in the shape of the IoT does not come without challenges. Compared to previous information and communication technologies, the IoT is much more complex and abstract. The introduction of smart devices to a network has created more information sources, increasing the amount of data gathered. Furthermore, the ability to communicate and act among themselves causes a shift from the user being in charge to a more mutual relationship between user and technologies. This information stream and communication contribute to new challenges faced by users: coping with a large amount of data, less autonomy because of the data-based decisions made by technology; less visibility and more ambiguity due to these decisions being made for the user; and greater risks regarding security and privacy issues (Van Deursen \& Mossberger, 2018).

From digital divide research - which traditionally studies differences in access to a technology - we know that among the most important determinants of using technology are user skills. In terms of skills, Web developments went from skills enabling operating and navigating within an online environment to more emphasis on skills involving information searches and interpretation, social interaction, and content creation. These skills can be linked to Web 1.0 and Web 2.0, respectively (Van Deursen, Helsper, Eynon, \& Van Dijk, 2017). What skills are needed to function in the IoT environment is unclear. However, all Internet skills are likely to remain important contributors, as they are a condition for capitalization of online opportunities, obtaining positive outcomes from these online opportunities, and for the process of access and information inequality (Livingstone \& Helsper, 2010; Van Deursen \& 
Mossberger, 2018; Van Dijk \& Van Deursen, 2014). Therefore, it is important to investigate what the effects of new Internet developments are in relation to Internet skills and technology acceptance.

Obtaining benefits from the IoT and coping with IoT challenges to a large extent depend on skills and informed use of the individual. Due to the changes in Internet technology, it is unclear what the skills to use the IoT comprise. In the current study, we examine to what extent Internet skills matter for the use of the IoT. We specifically focus on the role of Internet skills in relation to IoT technology acceptance, as IoT-related skills are expected to influence perceptions regarding its acceptance. This is important because despite associations with the Internet, the IoT has not been widely adopted yet. Therefore, the questions to ask are (1) to what extent Internet skills can be associated with skills needed to operate the IoT and (2) whether possessing these "IoT skills" contributes to greater technology acceptance and, thus, future usage of IoT. We aim to answer these questions through a survey with a sample representative of the Dutch population. This study is guided by a conceptual model that hypothesizes how the technology acceptance of the IoT is predicted by Internet and IoT skills.

\section{Theoretical background}

\subsection{Internet-of-Things Acceptance}

A model commonly used to investigate technology acceptance is the technology acceptance model (TAM; Davis, 1989). The TAM, derived from the theory of reasoned action (TRA; Fishbein \& Ajzen, 1975), is considered a leading model for explaining user acceptance of information technologies and is known for its robustness (Lee, Kozar, \& Larsen, 2003). The model has proven to be successful in explaining the acceptance of new technologies, including that of the IoT (e.g., Gao \& Bai, 2014; Karahoca, Karahoca, \& Aksöz, 2018; Lee \& Lee, 2018; Lekjaroen et al., 2016; Mital, Chang, Choudhary, Papa, \& Pani, 2017; Park, Cho, Han, \& Kwon, 2017; Patil, 2016). The TAM proposes that the beliefs on perceived usefulness (PU) and perceived ease of use (PEoU) influence one's attitude towards a technology, which, in turn, influences the actual technology use or intention to use it (in case the technology is not yet available). In addition to being directly associated with attitude, PEoU influences PU, and PU can be directly associated with the use of technology or the intention to do so. In this model, PU can be described as the extent to which someone believes that the technology will enhance their performance. PEoU refers to the extent to which someone believes that using the technology is free of effort (Davis, 1989).

The perceived (potential) advantages of the IoT compared to that of existing technologies can be illustrated by using the example of a smart thermostat. This "thing” enables close monitoring of energy consumption, and it can anticipate to your location by managing the room temperature upon leaving (by lowering the temperature) and returning home (by raising the temperature). If used adequately, these functions can be translated to both energy savings and improved living convenience, resulting in a high PU in terms of the TAM. Unlike PU, the autonomous character of changing room 
temperature seems to make PEoU subordinate. However, most IoT devices need initial setup. Personalizing the settings of the smart thermostat - not only considering location but also settings such as privacy - does make PEoU relevant for the acceptance of the IoT. In daily use, the importance of PEoU becomes apparent in, for example, the ongoing monitoring of energy consumption. Individuals should be able to read and make sense of the continuous collection of complex and ambiguous data. Often, it will be unclear what specific data are collected (and for what purpose) and who has access to it (Van Deursen \& Mossberger, 2018). This illustration, together with the previous application of the TAM in the context of the IoT, has led to the following hypotheses:

H1: $\quad$ IoT attitude is positively associated with IoT use.

H2: $\quad$ PU is positively associated with (a) IoT attitude and (b) IoT use.

H3: $\quad$ PEoU is positively associated with (a) IoT attitude and (b) PU.

\subsection{Internet-of-Things skills}

Unlike earlier technologies, operating IoT devices does not involve continuous interactions between users and devices. These devices operate rather autonomously using input from the environment via sensors (e.g., the smart thermostat). Therefore, people should be able to familiarize themselves with the enormous amount of data gathered (without their interference). This would involve scrutinizing device settings, in addition to data visualization, interpretation, and sharing. A clear visualization facilitates interpretation. Data sharing is required to, for example, compare data from other users, make sense of their comments and opinions, and discuss data with one another online or offline. This familiarization would enable strategic decision making on what data are collected by the IoT and how to address these data (e.g., choice of analysis, application, and communication) (Van Deursen \& Mossberger, 2018).

Next to the amount of data, the complexity of the data is increasing (Van Deursen \& Mossberger, 2018). This can be attributed to the ability of IoT systems to make data-based decisions without interference from the user. This autonomous decision making, together with the connectivity between users and other devices, can result in problems identifying who owns the data and defining system boundaries (Popescul \& Georgescu, 2013). Therefore, it will be hard to understand the exchange of information in the IoT system involving interactions with people and devices located in different contexts. This lack of understanding is subsequently reflected in difficulties in reasoning regarding devices' interactions and their effects, which, in turn, lead to incomplete specifications and misinterpretation. Lacking the skills to correctly interpret, analyze, and communicate data could result in users collecting irrelevant data, failing to apply the data, or drawing wrong conclusions. Take for example the case of a Dutch insurance company offering privileges when reaching a targeted number of (walking) steps. When an activity tracker is not calibrated properly, it could provide incorrect information. This information could result in the insurance company - which is connected to the same 
network - revoking privileges you could have earned when reaching the targeted number of steps. Even though you might have actually taken the targeted number of steps you would not benefit from it, as you did not notice the inaccuracies or failures in data collection and/or sharing of the data with the consequence of being overcharged. Considering this, we think of IoT skills as the skills to address smart devices, the so-called "things", and the data these devices gather. These skills include changing settings, interpreting data, sharing data with others and creating logical data visualizations to use smart devices to their full potential.

In relation to IoT acceptance, we expect IoT skills to influence both PU and PEoU, as skills are developed through experience (e.g., Agarwal \& Prasad, 1999; Dishaw \& Strong, 1999; Jackson, Chow, \& Leitch, 1997; Venkatesh, 2000; Venkatesh \& Morris, 2000). Internet skills develop through learning from doing, trial and error, problem sharing, and comparing to others (Van Dijk \& Van Deursen, 2014). Furthermore, skills are developed through experiencing what to pay attention to when problems occur (Harmon \& King, 1985; Jackson et al., 1997). Some studies focusing on the acceptance of the IoT included perceived behavioral control (PBC) (e.g., Gao \& Bai, 2014; Park et al., 2017; Patil, 2016) or, in other words, the users' assessment of skills, abilities, and resources to successfully perform IoTrelated tasks and behaviors (Park et al., 2017).

In addition to affecting PU and PEoU, we expect that IoT skills are positively associated with behavioral intention of using the new technology or, in case of early adoption, the use of the technology in question. Earlier studies showed that experience was positively associated with behavioral intention to use an information system (Jackson et al., 1997) and that PBC was positively associated with the intention of using the IoT (Gao \& Bai, 2014; Patil, 2016). Therefore, we hypothesize the following:

H4: $\quad$ IoT skills are positively associated with (a) PU, (b) PEoU, and (c) IoT use.

\subsection{Internet skills}

In contrast to IoT skills, there is a substantial body of literature regarding Internet skills. Based on the nature of Internet skills being transferable to new Web developments (Van Deursen \& Mossberger, 2018), we expect that they will remain relevant for the IoT. While originally most attention was on technical skills, in the past years, several skill dimensions or multifaceted conceptualizations have been addressed. A recent framework labelled Internet Skills Scale (ISS) proposed dimensions encompassing not only technical ability but also other skill types focusing on different engagements one can have with the Internet (Van Deursen, Helsper \& Eynon, 2016). The skill types covering the technical ability to use the Internet are operational skills and mobile skills. Information navigation skills comprise the ability to search the Internet, including finding, selecting, and evaluating information sources on the Internet. Together, operational, mobile and information navigation skills can be considered Web 1.0 skills, as they originally formed the basis of Web 1.0 activities. However, these skills have now been transferred to mobile devices. Mobile skills enable investigating the distribution of skills in using mobile 
devices, such as downloading and installing applications and monitoring the data costs involved in online mobile use (Van Deursen et al., 2016). In turn, these Web 1.0 skills are a requirement for Web 2.0 skills: social and creative skills. Social skills enable using online communication and interactions to understand and exchange meaning, involving searching, selecting, evaluating, and acting on online contacts. Creative skills are the skills necessary to create content suitable for online display. This content can include text, music and video, photo or image, multimedia or remixed media. Furthermore, creative skills also involve the basics of uploading this content (Van Deursen et al., 2016).

The skills proposed in the section above have a sequential and conditional nature: having operational, mobile and information navigation skills is related to the possession of social and creative skills (Van Deursen et al., 2017). We expect that IoT skills can be added to the skill dimensions in a similar manner. In the current contribution we first focus on mobile skills, as these are the most important subset of operational skills required for operating the IoT system. We expect that mobile skills will be needed for the initial setup and configurations of settings, but will play a less profound role in the continuous interaction between users and technology. In contrast, we expect information navigation, and especially social and creative skills to become more important for interaction in the IoT system due to the emphasis on content visualization, interpretation, and sharing. Together, these expectations lead to the following hypotheses:

H5: Mobile Internet skills are positively associated with (a) information navigation Internet skills, (b) social Internet skills, and (c) creative Internet skills.

H6: Information navigation Internet skills are positively associated with (a) social Internet skills and (b) creative Internet skills.

H7: $\quad$ Social Internet skills are positively associated with creative Internet skills.

H8: (a) Mobile Internet skills, (b) information navigation Internet skills, (c) social Internet skills, and (d) creative Internet skills are positively associated with IoT skills. 


\subsection{Conceptual model}

The conceptual model is presented in Figure 1. This model displays the hypothesized relations between the Internet skills, which are presented in the first block, IoT skills, and the TAM among both users and nonusers.

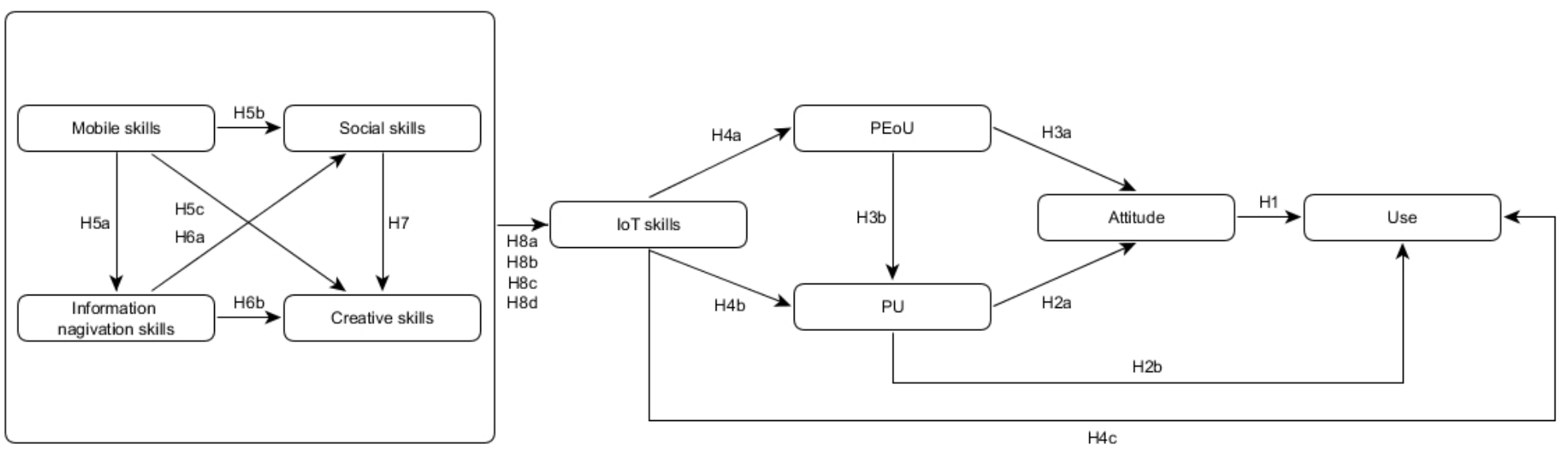

Figure 1. Conceptual model.

\section{Method}

\subsection{Sample}

In the present study, a representative sample of the Dutch population was recruited by using an online survey. To obtain this sample, we made use of PanelClix, a Dutch professional market research organization containing approximately 110,000 people. This panel is considered a representative sample of the Dutch population. We asked 1,698 individuals who participated in an Internet survey two weeks earlier to participate in a subsequent survey concerning the IoT. This would allow more space for IoTrelated questions, as important respondent characteristics and Internet skills were already covered in the first survey. During the original data collection, modifications were made to ensure that the sample was representative of the Dutch population. For the IoT survey, we aimed to obtain a sample of 1,200 respondents. Of the 1,698 contacted respondents, 1,356 participated in the IoT survey. In terms of gender, age, and educational level, the sample was consistent with official national statistics of the Dutch adult population. Table 1 summarizes the demographic profile of the respondents. The mean age of the respondents was 54.18 years $(\mathrm{SD}=18.07)$, ranging from 16 to 96 years.

The IoT survey was pilot tested with eight Internet users over two rounds. Modifications were made based on the feedback provided. In the second round, no major comments were provided. The time needed to answer the survey questions varied due to the number of IoT devices respondents used. On average, it took the respondents 25 minutes to complete the survey. 
Table 1. Demographic profile (N=1356).

\begin{tabular}{ccc}
\hline & $\mathrm{N}$ & $\%$ \\
\hline Gender & & \\
Male & 705 & 52 \\
Female & 651 & 48 \\
Age (years) & & \\
$16-25$ & 108 & 8 \\
$26-35$ & 169 & 12 \\
$36-45$ & 148 & 11 \\
$46-55$ & 239 & 18 \\
$56-65$ & 261 & 19 \\
$65-75$ & 247 & 18 \\
$75+$ & 184 & 14 \\
Education & & 33 \\
Low & 444 & 38 \\
Medium & 512 & 29 \\
High & 400 & \\
\hline
\end{tabular}

\subsection{Measures}

To measure IoT use, a distinction was made between users $(\mathrm{N}=563)$ and non-users $(\mathrm{N}=793)$ by asking respondents if they used one or more of an extensive list of 52 IoT devices that are available to consumers in the domains of health, home, and security $(\mathrm{M}=1.10, \mathrm{SD}=2.06)$. These domains were chosen based on their rapid development, facilitating widespread availability to consumers (Miorandi, Sicari, De Pellegrini, \& Chlamtac, 2012). When respondents own IoT devices but not use them as such, for example smart thermostats not connected to the internet, it is not measured as IoT use. For the other TAM constructs, we used an adapted version of the original instrument. We conducted an exploratory factor analysis with varimax rotation for $P U, P E o U$, and IoT attitude. Factor loadings were employed at .40 and above. In total, 26 of 27 items were retained. On behalf of the internal consistency, one additional item was removed. We retained ten items for PU, five for PEoU, and ten for IoT attitude. The items were answered using a 5-point Likert scale ranging from (1) ‘Disagree', to (5) ‘Agree'. Cronbach's $\alpha$ was .90 for PU, .71 for PEoU, and .88 for attitude. For an overview of the items, we refer to Appendix A.

IoT skills were measured using a set of items constructed for the purpose of this study. This set initially consisted of 11 statements covering participants' knowledge regarding how to address smart devices and the information they gather. Two negatively stated items (reversed) were removed after conducting an exploratory factor analysis. The remaining 9 items loaded on one factor, together having an internal reliability of .96. Thus, for this first empirical investigation of IoT skills, we consider IoT skills as a single variable covering a wider set of skills. To respond to the items, a 6-point Likert-type 
scale was used, which ranged from one, 'not at all true for me', to five, 'very true for me', with 'neither true nor untrue for me' as the neutral response (Van Deursen et al., 2016). When respondents did not understand the item, they could respond by using zero, 'I don't understand this statement'. See Appendix A for all items.

To measure Internet skills, we used items on mobile, information navigation, social, and creative skills from the Internet Skills Scale (ISS) (Van Deursen et al., 2016). As mobile skills were treated as the skills needed to operate the IoT system, operational skills were excluded from the current study. In total, 18 items were used from the original ISS: five items for navigation information skills, five for social skills, and five for creative skills. We used the three ISS items for mobile skills and added three additional items. We used the same Likert-type scale as for IoT skills. In Appendix A, an overview of all 21 items can be found with the descriptive statistics. Scores on the different skill types exhibited sufficient internal consistency. Cronbach's $\alpha$ was .77 for information navigation skills, .86 for social skills, .85 for creative skills, and .89 for mobile skills.

\subsection{Analysis}

To test our hypotheses presented in the conceptual model shown in Figure 1, we applied path analysis using Amos 23.0. Missing values were replaced by the series mean, as the maximum number of values missing on an item did not exceed 17. To obtain comprehensive model fit, we included the $\chi^{2}$ statistic, the ratio of $\chi^{2}$ to its degree of freedom ( $\chi^{2} / \mathrm{df}$ ), the standardized root mean residual (SRMR), the TuckerLewis index (TLI), and the root mean square error of approximation (RMSEA).

\section{Results}

\subsection{Structural and path model}

All basic assumptions for structural equation modeling were met. Hoelter's critical $\mathrm{N}$ was used to decide whether the sample size was adequate for applying structural equational modeling. The model, as presented in Figure 2, resulted in a Hoelter's N of 349 at the .05 level of significance, and 421 at the .01 levels of significance. Therefore, the sample size was sufficient, as Hoelter's N > 200 (Hoelter, 1983). The fit results obtained from testing the validity of a causal structure of the conceptual model are as follows: $\chi^{2}(18)=112.22 ; \chi^{2} / \mathrm{df}=6.23$; SRMR $=.04$; TLI $=.93$; RMSEA $=.06$ (90\% confidence interval $[\mathrm{CI}]=.05, .07)$. The model explains $18 \%$ of the variance in IoT use. Table 2 provides the correlations between the variables. Figure 2 provides the path models with coefficients and variances explained.

Table 2. Correlation matrix.

\begin{tabular}{|c|c|c|c|c|c|c|c|c|c|}
\hline & 1 & 2 & 3 & 4 & 5 & 6 & 7 & 8 & 9 \\
\hline 1. Mobile skills & - & .27 & .47 & .43 & .55 & .21 & .36 & .13 & .28 \\
\hline
\end{tabular}




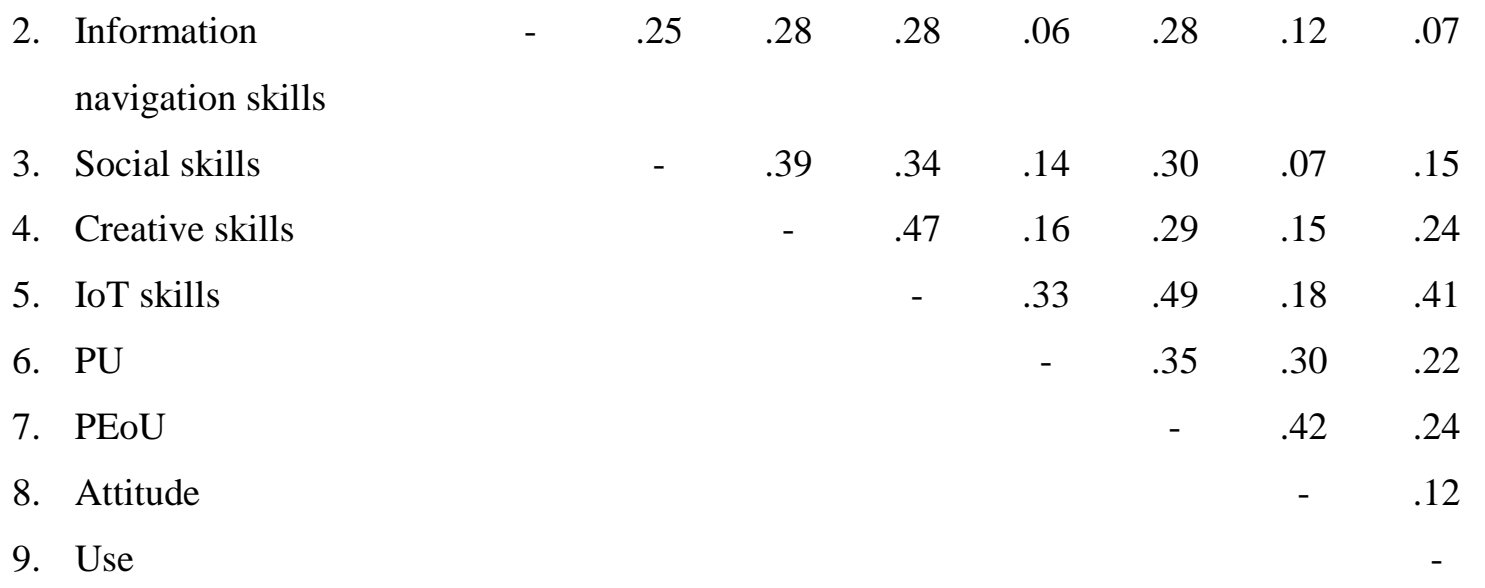

Note: numbers displayed are significant at p-value $<.05$.

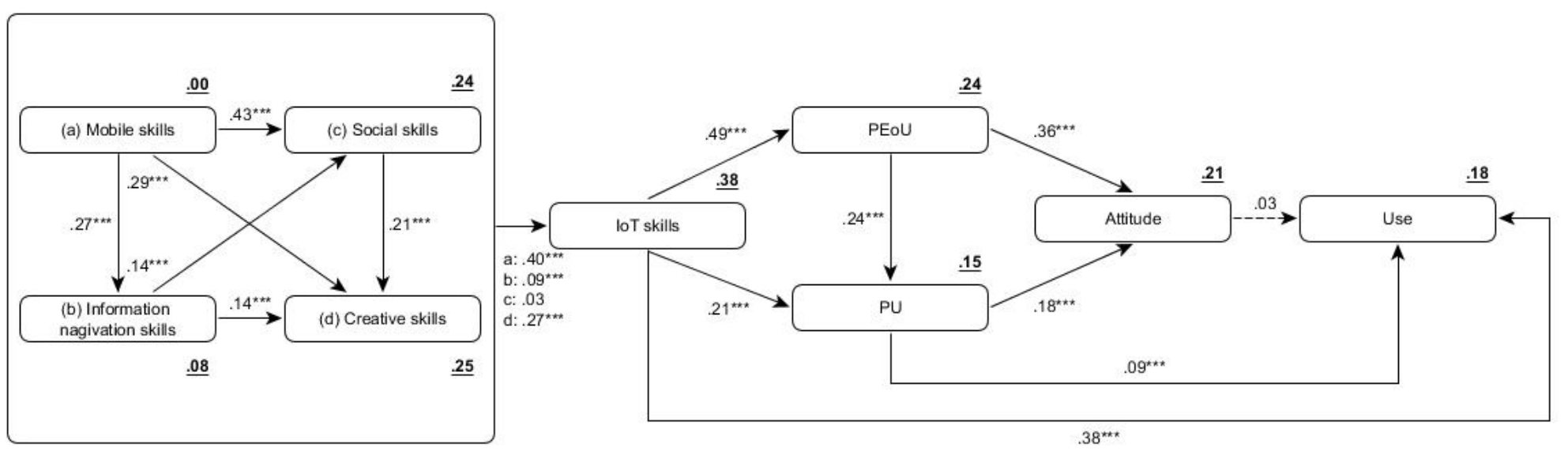

Figure 2. Results for the research model with path coefficients. Note: ${ }^{*} \mathrm{p}<.05 ;{ }^{* *} \mathrm{p}<.01 ;{ }^{* * *} \mathrm{p}<.001$ level. The dotted lines are non-significant paths. Squared multiple correlations are underlined.

\subsection{Overview of the hypotheses}

The standardized path coefficients in Figure 2 show various significant direct and indirect effects among Internet skills, IoT skills, perceptions on the IoT, and use. Table 3 summarizes the hypotheses. Except for two, all hypotheses are supported. Despite the (partial) rejection of two hypotheses, the proposed model provides an adequate explanation for the use of IoT.

The first hypothesis (H1) suggesting that a positive attitude positively influences IoT use is rejected. However, the other hypotheses regarding the TAM could be confirmed. PU is found to positively influence attitude (H2a) and IoT use (H2b), and H3 is supported in its entirely. PEoU influences attitude both directly and indirectly (H3a), and it is found to directly influence PU (H3b). Furthermore, H4 could also be confirmed, as PU and IoT use are both influenced directly and indirectly by IoT skills (H4a and H4c, respectively), and the IoT directly influences PEoU (H4b).

All the hypotheses regarding the relations between the different types of Internet skills are supported. Following the sequential character of Internet skills, mobile skills directly influence information navigation skills (H5a), and influence social (H5b) and creative skills (H5c) both directly 
and indirectly. This sequentiality is also supported for information navigation skills, as they directly influence social skills (H6a) and creative skills (H6b). Besides the direct influence of information navigation skills on creative skills, an indirect influence is found. Furthermore, social skills influence creative skills (H7), completing the sequence of Internet skills.

In addition to the relations between Internet skills, Table 3 shows the relationships between the Internet skills and IoT skills. Following the conceptual model, hypotheses H8a and H8b are both supported, as mobile and information navigation skills influence IoT skills directly and indirectly. Furthermore, creative skills are found to directly influence IoT skills, confirming hypothesis H8d. In turn, this influence of creative skills on IoT skills has led to the partial support of H8c, as social skills could only be indirectly associated with IoT skills through creative skills.

Table 3. Significant direct, indirect, and total effects of IoT skills on technology acceptance and of Internet skills on IoT skills.

\begin{tabular}{lcccc}
\hline Link & $\begin{array}{c}\text { Direct } \\
\text { effects } \beta\end{array}$ & $\begin{array}{c}\text { Indirect } \\
\text { effects } \beta\end{array}$ & $\begin{array}{c}\text { Total } \\
\text { effects } \beta\end{array}$ & Validation \\
\hline H1. Attitude - IoT use & - & - & - & Rejected \\
H2a. PU - Attitude & .18 & - & .18 & Supported \\
H2b. PU - IoT use & .09 & .00 & .09 & Supported \\
H3a. PEoU - Attitude & .36 & .04 & .40 & Supported \\
H3b. PEoU - PU & .24 & - & .24 & Supported \\
H4a. IoT skills - PU & .21 & .12 & .33 & Supported \\
H4b. IoT Skills - PEoU & .49 & - & .49 & Supported \\
H4c. IoT skills - IoT use & .38 & .04 & .44 & Supported \\
H5a. Mobile skills - Information navigation skills & .27 & - & .27 & Supported \\
H5b. Mobile skills - Social skills & .43 & .04 & .47 & Supported \\
H5c. Mobile skills - Creative skills & .29 & .14 & .43 & Supported \\
H6a. Information navigation skills - Social skills & .14 & - & .14 & Supported \\
H6b. Information navigation skills - Creative skills & .14 & .03 & .17 & Supported \\
H7. Social skills - Creative skills & .21 & - & .21 & Supported \\
H8a. Mobile skills - IoT skills & .40 & .15 & .55 & Supported \\
H8b. Information navigation skills - IoT skills & .09 & .05 & .14 & Supported \\
H8c. Social skills - IoT skills & - & .06 & .06 & Partly \\
& .27 & - & .27 & Supported \\
H8d. Creative skills - IoT skills & & & & sipuported \\
\hline Note: effects are significant at p < 05 level & & & &
\end{tabular}

Note: effects are significant at $\mathrm{p}<.05$ level. 


\section{Discussion}

\subsection{Main findings}

Concerning the first research question - to what extent can Internet skills be associated with skills needed to operate the IoT - we can conclude that Internet skills are important for performing on IoT skills. Three out of the four Internet skill types, namely, mobile, information navigation, and creative skills, directly contribute to possessing IoT skills. Additionally, mobile, information navigation, and social skills indirectly contribute to IoT skills through creative skills. Together, these findings suggest that the skills needed to use the IoT, to a certain extent, depend on the Internet skills from previous Web developments. Prior research on Internet skills reveals that for large parts of the population there is much room for improvement. Especially for information navigation, social, and creative skills, many users still struggle, resulting in less than optimal Internet usage (Van Deursen \& Helsper, 2017). As these skills appear important for using IoT, skill improvements are likely to be needed in Web 4.0 environments. Following the same line of reasoning, prior research also found that traditional literacy skills (i.e., reading, writing, and understanding texts) are important for Internet performance (Van Deursen \& Van Dijk, 2016). Being skilled in using the IoT requires sufficient Internet skills and sufficient levels of traditional literacy. Despite the autonomous power of the IoT, these skills do not magically disappear.

The lack of a direct link between social and IoT skills could indicate unawareness of the social skills that are applied in an IoT context. Due to the autonomous character of the IoT, social skills might have moved to the background. Sharing content has become a matter of making choices regarding changing settings of what to share, when to share, and who to share with. Nonetheless, knowledge on the settings regarding data sharing is required since the collected data comprise personal information on habits and health, which could also be of interest to others (Van Deursen \& Mossberger, 2018). For example, you are keeping track of your exercising behavior, and you are going for the same run every Wednesday. Your weekly exercise is automatically shared with your trainer to keep track of your progress, but you do not want to publicly share every time you go for a run, as it reveals when nobody is home, which could make you vulnerable to burglary. The need for consideration of such potential consequences of data sharing behavior (together with having to change sharing settings to your personal preferences) underlines the importance of awareness regarding autonomous content sharing and strategic decision making. Developers of the IoT should provide guidance by clarifying what data are gathered, with whom the data are shared, and how to change settings related to data sharing. In anyway, social skills are also important in the IoT. Furthermore, social skills related to Web 2.0 remain important as also in IoT contexts, it is possible to share collected content via the 'traditional' Internet. One could for example share data when having set a personal record at a running competition. 
Regarding the second research question, we can conclude that IoT skills contribute to the use of the IoT. IoT skills contribute both directly and indirectly, indicating that people's assessments of their own capabilities in using the IoT are important for actually starting to use the IoT. Moreover, their assessment form a base for deciding how easy it is to use the technology in place and how useful it can be to them (Gong, Xu, \& Yu, 2004; Teo, 2009). The results only show a direct influence of PU on IoT use and an indirect influence of PEoU on IoT use through PU. Attitude towards the IoT does not affect use. This finding indicates that people are motivated to use the IoT because it is useful to them, even though they do not have an outspoken positive attitude towards using it. Such pragmatic mindset might relate to the IoT being a relatively new technology that is continuously developing. People still need to form an opinion towards it: they can see the direct benefits of using devices connected to the IoT (PU), but they have not thought of or do not fully understand the possible implications this use can have for their lives or for society. In line with this argument, the overall responses on IoT attitude were relatively neutral, which could indicate both neutrality and indecisiveness (Croasmun \& Ostrom, 2011).

When looking at the specific items making up IoT attitude, we did not expect people to have a neutral attitude towards privacy. We anticipated an increase in data security concerns, as Web 2.0 is being negatively reported on in the media following social network crimes involving personal private information (Ortiz, Chih, \& Tsai, 2018). This is worrisome, as the increased monitoring and data collection of the IoT generates large amounts of personal and sensitive data, which we should treat with care. The observed neutrality in this study might suggest that potential risks are overlooked by users, as the IoT operates in the background. Data collection, analysis, data usage, and sharing are often not transparent, making it more difficult to make strategic decisions (Van Deursen \& Mossberger, 2018). Although we did not account for privacy and security constructs as potential predictors in the current contribution, the user skill perspective implicitly addressed related concerns. For example, privacy decision making was incorporated in both the constructs of IoT skills and attitude. As this study was designed to address and stress the role of IoT skills, future studies might incorporate privacy and security concerns separately to see how their contribution compares to the importance of skills.

\subsection{Limitations}

This study has several limitations that need to be addressed. IoT skills were treated as a single variable, as all items loaded on one construct. However, through the development of the Web, Internet skills have been proven to consist of multiple dimensions, for example, the ones covered in the current study. Although IoT skills might be the Web 4.0 addition to Internet skills (in a similar manner as social and content creation were added in the Web 2.0 era), we belief that the complexity of the IoT system represents different skills needed to successfully use the IoT. Future investigations might try to further address the different underlying skill facets. One example of such skill facet could be that of data literacy skills (Pangrazio \& Selwyn, 2018). Nevertheless, we have shown that all Internet skills remain important for performing on IoT skills. 
The conceptual model used to study the effect of skills on the acceptance of the IoT was based on the TAM concept. Besides its robustness, the TAM was chosen for its simplicity. The skill related concepts of PU and PEoU make the TAM a perfect fit for the main focus of this study: investigating the role of user skills. Furthermore, by adding IoT skills to the TAM we addressed the criticism of overreliance on PU of PEoU and neglecting other factors (Lee et al., 2003). However, for further research on IoT acceptance we recommend to extend the TAM to suit the characteristics of IoT, as extensions have been proven successful for other technological developments such as cloud computing (Park \& Kim, 2014). Studies that are interested in adding additional explanations might also take the TAM2 (Venkatesh \& Davis, 2000) or the Unified Theory of Acceptance and Use of Technology (UTAUT; Venkatesh, Morris, Davis, \& Davis, 2003) as point of departure. The limited tailoring of the TAM to the IoT also shows in the manner IoT acceptance was addressed in this study. Originally, the TAM was applied to specific tasks, whereas we used it to study IoT acceptance in its generality. As a lack of focus has caused mixed results in technology perceptions (Dishaw \& Strong, 1999; Lee et al., 2003), this could be another reason why attitude does not influence the use of the IoT. In future studies, we therefore recommend focusing on one particular application of an IoT device.

Similar to most TAM studies, this study makes use of self-reporting. However, this is not always an accurate measure, as self-reported use does not necessarily correspond with actual use (Legris, Ingham, \& Collerette, 2003). People might, for example, not be aware that the smart technologies they use are part of the IoT system or might not even know they participate in this network of things due to its ubiquitous and autonomous character. On the other hand, people might consider smart devices the IoT (while they are not), or they might not use the devices as such. Additionally, for measuring Internet skills, self-reporting is not the most accurate measure. People tend to underestimate or overestimate their level of skills (Hargittai, 2005; Merritt, Smith, \& Di Renzo, 2005; Van Deursen \& Van Dijk, 2010). However, despite the inaccuracy of self-reporting, the results of this study are suitable to serve as an indicator and provide us with a starting point to objectively study what skills are needed to use the IoT, for instance, by conducting performance tests.

Finally, the generalizability of results should be considered. As the current study was conducted in the Netherlands, a country with high household Internet penetration, results on skills and acceptance could deviate from other countries of which Internet penetration is still growing. The Netherlands can be seen as a forerunner of trends and is therefore considered appropriate for research on the necessity of (Internet) skills to operate IoT and, in the distant future, the distribution of skills among the general population. 


\section{Acknowledgement}

This work is part of the research programme 'Any Thing for Anyone? An individual and sociocontextual approach to Internet-of-Things skill inequalities' with project number 452-17-001, which is (partly) financed by the Netherlands Organisation for Scientific Research (NWO).

\section{References}

Agarwal, R., \& Prasad, J. (1999). Are Individual Differences Germane to the Acceptance of New Information Technologies? Decision Sciences, 30(2), 361-391. https://doi.org/10.1111/j.15405915.1999.tb01614.x

Atzori, L., Iera, A., \& Morabito, G. (2010). The Internet of Things: A survey. Computer Networks, 54(15), 2787-2805. https://doi.org/10.1016/j.comnet.2010.05.010

Croasmun, J. T., \& Ostrom, L. (2011). Using Likert-Type Scales in the Social Sciences. Journal of Adult Education, 40(1), 19-22.

Davis, F. D. (1989). Perceived Usefulness, Perceived Ease of Use, and User Acceptance of Information Technology. MIS Quarterly, 13(3), 319-340. https://doi.org/10.2307/249008

Dishaw, M. T., \& Strong, D. M. (1999). Extending the technology acceptance model with tasktechnology fit constructs. Information \& Management, 36(1), 9-21. https://doi.org/10.1016/S0378-7206(98)00101-3

Fishbein, M. A., \& Ajzen, I. (1975). Belief, attitude, intention and behaviour: An introduction to theory and research. Reading, MA: Addison-Wesley.

Gao, L., \& Bai, X. (2014). A unified perspective on the factors influencing consumer acceptance of internet of things technology. Asia Pacific Journal of Marketing and Logistics, 26(2), 211-231. https://doi.org/10.1108/APJML-06-2013-0061

Gong, M., Xu, Y., \& Yu, Y. (2004). An Enhanced Technology Acceptance Model for Web-Based Learning. Journal of Information Systems Education, 15(4), 365-374.

Gubbi, J., Buyya, R., Marusic, S., \& Palaniswami, M. (2013). Internet of Things (IoT): A Vision, Architectural Elements, and Future Directions. Computer Networks, 29(7), 1645-1660. https://doi.org/10.1016/j.future.2013.01.010

Hargittai, E. (2005). Survey Measures of Web-Oriented Digital Literacy. Social Science Computer Review, 23(3), 371-379. https://doi.org/10.1177/0894439305275911

Harmon, P., \& King, D. (1985). Expert systems: Artificial intelligence in business. New York: John 
Wiley, Inc.

Hoelter, J. W. (1983). The Analysis of Covariance Structures. Sociological Methods \& Research, 11(3), 325-344. https://doi.org/10.1177/0049124183011003003

Jackson, C. M., Chow, S., \& Leitch, R. A. (1997). Toward an Understanding of the Behavioral Intention to Use an Information System. Decision Sciences, 28(2), 357-389. https://doi.org/10.1111/j.1540-5915.1997.tb01315.x

Karahoca, A., Karahoca, D., \& Aksöz, M. (2018). Examining intention to adopt to internet of things in healthcare technology products. Kybernetes, 47(4), 742-770. https://doi.org/10.1108/K-02-20170045

Lee, S.-B., \& Lee, W.-H. (2018). An Empirical Study on the Effect of Bundling Service on User Acceptance of IoT Services. Journal of Theoretical and Applied Information Technology, 96(6), 1701-1710.

Lee, Y., Kozar, K. A., \& Larsen, K. R. T. (2003). The Technology Acceptance Model: Past, Present, and Future. Communications of the Association for Information Systems, 12(1), 752-780.

Legris, P., Ingham, J., \& Collerette, P. (2003). Why do people use information technology? A critical review of the technology acceptance model. Information \& Management, 40(3), 191-204. https://doi.org/10.1016/S0378-7206(01)00143-4

Lekjaroen, K., Ponganantayotin, R., Charoenrat, A., Funilkul, S., Supasitthimethee, U., \& Triyason, T. (2016). IoT Planting: Watering system using mobile application for the elderly. In 2016 International Computer Science and Engineering Conference (ICSEC) (pp. 1-6). IEEE. https://doi.org/10.1109/ICSEC.2016.7859873

Livingstone, S., \& Helsper, E. J. (2010). Balancing opportunities and risks in teenagers' use of the internet: the role of online skills and internet self-efficacy. New Media \& Society, 12(2), 309329. https://doi.org/10.1177/1461444809342697

Merritt, K., Smith, K. D., \& Di Renzo, J. C. (2005). An Investigation of Self-Reported Computer Literacy: Is it Reliable? Issues in Information Systems, 6(1), 289-295.

Miorandi, D., Sicari, S., De Pellegrini, F., \& Chlamtac, I. (2012). Internet of things: Vision, applications and research challenges. Ad Hoc Networks, 10(7), 1497-1516. https://doi.org/10.1016/j.adhoc.2012.02.016

Mital, M., Chang, V., Choudhary, P., Papa, A., \& Pani, A. K. (2017). Adoption of Internet of Things in India: A test of competing models using a structured equation modeling approach. Technological Forecasting \& Social Change. https://doi.org/10.1016/j.techfore.2017.03.001 
Noh, Y. (2015). Imagining Library 4.0: Creating a Model for Future Libraries. The Journal of Academic Librarianship, 41(6), 786-797. https://doi.org/10.1016/j.acalib.2015.08.020

Ortiz, J., Chih, W.-H., \& Tsai, F.-S. (2018). Information privacy, consumer alienation, and lurking behavior in social networking sites. Computers in Human Behavior, 80, 143-157. https://doi.org/10.1016/j.chb.2017.11.005

Pangrazio, L., \& Selwyn, N. (2018). "Personal data literacies”: A critical literacies approach to enhancing understandings of personal digital data. New Media \& Society, 1-19. https://doi.org/10.1177/1461444818799523

Park, E., Cho, Y., Han, J., \& Kwon, S. J. (2017). Comprehensive Approaches to User Acceptance of Internet of Things in a Smart Home Environment. IEEE Internet of Things Journal, 4(6), 23422350. https://doi.org/10.1109/JIOT.2017.2750765

Park, E., \& Kim, K. J. (2014). An Integrated Adoption Model of Mobile Cloud Services: Exploration of Key Determinants and Extension of Technology Acceptance Model. Telematics and Informatics, 31(3), 376-385. https://doi.org/10.1016/J.TELE.2013.11.008

Patil, K. (2016). Retail adoption of Internet of Things: Applying TAM model. In 2016 International Conference on Computing, Analytics and Security Trends (CAST) (pp. 404-409). IEEE. https://doi.org/10.1109/CAST.2016.7915003

Perera, C., Zaslavsky, A., Christen, P., \& Georgakopoulos, D. (2014). Context Aware Computing for The Internet of Things: A Survey. IEEE Communications Surveys \& Tutorials, 16(1), 414-454. https://doi.org/10.1109/SURV.2013.042313.00197

Popescul, D., \& Georgescu, M. (2013). Internet-of-Things - Some Ethical Issues. The USV Annals of Economics and Public Administration, 13(2), 208-214.

Teo, T. (2009). Modelling technology acceptance in education: A study of pre-service teachers. Computers \& Education, 52(2), 302-312. https://doi.org/10.1016/J.COMPEDU.2008.08.006

Van Deursen, A. J. A. M., \& Helsper, E. J. (2017). Collateral benefits of Internet use: Explaining the diverse outcomes of engaging with the Internet. New Media \& Society, 1-19. https://doi.org/10.1177/1461444817715282

Van Deursen, A. J. A. M., Helsper, E. J., \& Eynon, R. (2016). Development and validation of the Internet Skills Scale (ISS). Information, Communication \& Society, 19(6), 804-823. https://doi.org/10.1080/1369118X.2015.1078834

Van Deursen, A. J. A. M., Helsper, E. J., Eynon, R., \& Van Dijk, J. A. G. M. (2017). The Compoundness and Sequentiality of Digital Inequality. International Journal of Communication, 
$11,452-473$.

Van Deursen, A. J. A. M., \& Mossberger, K. (2018). Any Thing for Anyone? A New Digital Divide in Internet-of-Things Skills. Policy \& Internet, 10(2), 122-140. https://doi.org/10.1002/poi3.171

Van Deursen, A. J. A. M., \& Van Dijk, J. A. G. M. (2010). Measuring Internet Skills. International Journal of Human-Computer Interaction, 26(10), 891-916. https://doi.org/10.1080/10447318.2010.496338

Van Deursen, A. J. A. M., \& Van Dijk, J. A. G. M. (2016). Modeling Traditional Literacy, Internet Skills and Internet Usage: An Empirical Study. Interacting with Computers, 28(1), 13-26. https://doi.org/10.1093/iwc/iwu027

Van Dijk, J. A. G. M., \& Van Deursen, A. J. A. M. (2014). Digital skills, unlocking the information society. NY: Palgrave Macmillan.

Venkatesh, V. (2000). Determinants of Perceived Ease of Use: Integrating Control, Intrinsic Motivation, and Emotion into the Technology Acceptance Model. Information Systems Research, 11(4), 342-365. https://doi.org/10.1287/isre.11.4.342.11872

Venkatesh, V., \& Davis, F. D. (2000). A Theoretical Extension of the Technology Acceptance Model: Four Longitudinal Field Studies. Management Science, 46(2), 186-204. https://doi.org/10.1287/mnsc.46.2.186.11926

Venkatesh, V., \& Morris, M. G. (2000). Why Don’t Men Ever Stop to Ask for Directions? Gender, Social Influence, and Their Role in Technology Acceptance and Usage Behavior. MIS Quarterly, 24(1), 115-139. https://doi.org/10.2307/3250981

Venkatesh, V., Morris, M. G., Davis, G. B., \& Davis, F. D. (2003). User Acceptance of Information Technology: Toward a Unified View. Source: MIS Quarterly, 27(3), 425-478. https://doi.org/10.2307/30036540

Whitmore, A., Agarwal, A., \& Da Xu, L. (2015). The Internet of Things-A survey of topics and trends. Information Systems Frontiers, 17(2), 261-274. https://doi.org/10.1007/s10796-014$9489-2$ 


\section{Appendix A}

\begin{tabular}{|c|c|c|}
\hline TAM & $\mathrm{M}$ & $\mathrm{SD}$ \\
\hline \multicolumn{3}{|l|}{ Perceived usefulness (PU) $(\alpha=.90)$} \\
\hline \multicolumn{3}{|l|}{ Using the Internet-of-Things... } \\
\hline ... improves living standards & 3.27 & 1.03 \\
\hline ... gives me freedom & 3.18 & .96 \\
\hline$\ldots$ is good for society & 3.02 & .96 \\
\hline$\ldots$ is good for humans & 2.98 & .92 \\
\hline ... improves my productivity & 3.14 & 1.06 \\
\hline ... makes life less burdensome & 3.27 & .92 \\
\hline ... makes life easier & 3.65 & .92 \\
\hline ... supports daily living & 3.64 & 1.00 \\
\hline ... makes life quicker & 3.71 & .94 \\
\hline ... makes life safer & 2.99 & .99 \\
\hline ... is good for the environment & 2.92 & .89 \\
\hline \multicolumn{3}{|l|}{ Perceived ease of use (PEoU) $(\alpha=.71)$} \\
\hline \multicolumn{3}{|l|}{ Using the Internet-of-Things... } \\
\hline$\ldots$ is unclear $(\mathrm{R})$ & 3.19 & .92 \\
\hline ... makes me feel uncomfortable because I don’t understand it (R) & 3.45 & 1.12 \\
\hline ... is easy to learn & 3.55 & .93 \\
\hline ... makes it hard to change settings to personal preferences (R) & 3.10 & .93 \\
\hline ... does not require much mental effort & 3.20 & .91 \\
\hline \multicolumn{3}{|l|}{ Attitude $(\alpha=.88)$} \\
\hline \multicolumn{3}{|l|}{ Using the Internet-of-Things... } \\
\hline ... makes life less social $(\mathrm{R})$ & 2.41 & 1.05 \\
\hline ... makes people servants of technology (R) & 2.35 & .97 \\
\hline ... will control our lives (R) & 2.42 & 1.02 \\
\hline ... makes people too dependent $(\mathrm{R})$ & 2.50 & .99 \\
\hline$\ldots$ is inflicted on us (R) & 2.60 & 1.05 \\
\hline ... dehumanizes society $(\mathrm{R})$ & 2.69 & 1.01 \\
\hline ... disrupts society $(\mathrm{R})$ & 3.05 & .99 \\
\hline ... makes it difficult to protect my privacy (R) & 2.34 & .98 \\
\hline ... causes mental instability $(\mathrm{R})$ & 3.02 & .93 \\
\hline$\ldots$ is humiliating to humans $(\mathrm{R})$ & 3.57 & 1.01 \\
\hline IoT skills & $\mathrm{M}$ & SD \\
\hline
\end{tabular}

IoT skills $(\alpha=.96)$ 
I know how to connect smart devices to the Internet

I know how to share information from smart devices on the Internet

I know how to operate smart devices by using applications

I know how to interpret data from smart devices

I know how to connect smart devices to my WiFi network

I find it hard to decide on the best settings for smart devices (R)

I feel confident operating smart devices

I find the way smart devices operate unclear $(R)$

I know how to change with whom I share data on a smart device

I know how to read data from smart devices

I know how to change how often data are gathered by smart devices

Information navigation skills $(\alpha=.77)$

I find it hard to decide what the best keywords are to use for online searches

I get tired when looking for information online

Sometimes I end up on Websites without knowing how I got there

I find it hard to find a Website I visited before

The way in which many Websites are designed confusing

Social skills $(\alpha=.86)$

I know how to change who I share content with (e.g. friends, friends of

friends or public)

I know how to remove friends from my contact lists

I am careful to make my comments and behaviors appropriate to the

situation I find myself in online

I know which information I should and shouldn't share online

I know when I should and I shouldn't share information online

Creative skills $(\alpha=.85)$

I know how to create something new from existing online images, music or video

I know how to make basic changes to the content that others have produced

I know how to design a Website

I know which different types of licenses apply to online content

I would feel confident putting video content I have created online

Mobile skills $(\alpha=.89)$

I know how to install apps on a mobile device 
I know how to switch off the location on my mobile device

I know how to upload a photo or video from my mobile device to social media

I know how to compare mobile apps to choose the best option 